Revue de l'Institut des langues et cultures

d'Europe, Amérique, Afrique, Asie et Australie

$34 \mid 2019$

Femmes et migrations aux $\mathrm{XIX}^{\mathrm{e}}$ et $\mathrm{XX} \mathrm{X}^{\mathrm{e}}$ siècles :

regards et représentations

\title{
Exploration des stratégies d'après-guerre. Destins croisés de deux Polonaises en migration. France (1945-1950)
}

Exploring Post-War Strategies. Intertwined Destinies of Two Polish Women in Migration. France 1945-1950

\section{Laurence Prempain}

\section{OpenEdition \\ Journals}

Édition électronique

URL : http://journals.openedition.org/ilcea/5928

DOI : 10.4000/ilcea.5928

ISSN : 2101-0609

Éditeur

UGA Éditions/Université Grenoble Alpes

\section{Édition imprimée}

ISBN : 978-2-37747-074-7

ISSN : 1639-6073

\section{Référence électronique}

Laurence Prempain, «Exploration des stratégies d'après-guerre. Destins croisés de deux Polonaises en migration. France (1945-1950) », ILCEA [En ligne], 34 | 2019, mis en ligne le 15 janvier 2019,

consulté le 10 décembre 2020. URL : http://journals.openedition.org/ilcea/5928 ; DOI : https://doi.org/ 10.4000/ilcea.5928

Ce document a été généré automatiquement le 10 décembre 2020.

(c) ILCEA 


\title{
Exploration des stratégies d'après- guerre. Destins croisés de deux Polonaises en migration. France (1945-1950)
}

\author{
Exploring Post-War Strategies. Intertwined Destinies of Two Polish Women in
}

Migration. France 1945-1950

Laurence Prempain

\section{Introduction}

1 1945. Lyon. Le monde sort progressivement de la guerre. Les rapatriéees des camps de détention, de concentration et d'extermination arrivent progressivement en France. Parmi eux, Irena Borsukowska. C'est la première fois qu'elle vient en France; elle y est autorisée car, jeune mariée, elle accompagne son époux, François Privey, de nationalité française, ancien déporté car n'ayant pas répondu à la mobilisation pour le $\mathrm{STO}^{1}$ et rencontré en Allemagne où elle-même a été déportée avec ses parents. Son regard croise peut-être celui d'Aldona Starzynska, qui guette les convois de rapatriéees. Elle attend son mari, Andrzej Starzynski. Andrzej Starzynski a combattu dans les rangs de l'armée polonaise en France, puis dans ceux de la Résistance polonaise en France, jusqu'à son arrestation et sa déportation en 1943. Aldona surveille les trains de rapatriement, mais son mari ne descendra d'aucun. Elle reste veuve avec ses deux filles, dont l'une n'a pas connu son père.

2 2016-2018. Élisabeth Privey, Wanda Starzynska-Aldono, deux femmes, deux rencontres ${ }^{2}$. Nées en 1944 et 1946, chacune porte à sa manière l'histoire de sa mère, Irena Privey, née Borsukowska pour la première, Aldona Starzynska, née Mikulski pour la seconde. Leurs mères sont nées polonaises au début $\mathrm{du} \mathrm{Xx}^{\mathrm{e}}$ siècle, ont vécu la Révolution russe, l'ont fuie alors enfant ou nourrisson pour rejoindre une Pologne renaissante ${ }^{3}$, puis ont vécu un 
temps plus ou moins long à Varsovie avant que, par des époques et des chemins bien différents, elles ne viennent en France et y restent. C'est à Lyon qu'elles se rencontrent, que leurs enfants jouent ensemble, restent plus ou moins liés. Histoires de femmes en migration, recueillies par l'auteure elle-même en migration, héritière d'une histoire en quelques points similaires. Une mise en abîme qui n'empêche pas de faire sien le point de vue de nos figures tutélaires ${ }^{4}:$ il y a nécessité de savoir d'où l'on parle. La question est donc de comprendre, dans la mesure du possible, quels mécanismes intellectuels ont conduit au choix d'un sujet de recherche ${ }^{5}$. Mon statut d'étrangère en Finlande a produit un écho, a transcendé le temps et les expériences et a forgé notamment cette question: quelles stratégies de vie deux jeunes femmes de nationalité polonaise installées en France ont-elles été obligées de développer pour faire face à leur isolement à la sortie de la Seconde Guerre mondiale ? Devenues amies, qu'est-ce qui rapproche la jeune veuve de la jeune mariée, elle aussi membre de l'intelligentsia polonaise mais qui a pourtant épousé un cheminot français, sur les recommandations de son père? Solidarité féminine, solidarité de classe, solidarité de l'exil ? Comment ont-elles vécu cette vie que je considère, à tort ou à raison, ne pas être le fruit de leur décision? Quelles identités se sont-elles construites?

3 Dans le cadre de cette contribution, après avoir présenté les cadres méthodologiques de cette recherche, je retracerai le parcours de vie d'Aldona Starzynska d'une part et d'Irena Borsukowska d'autre part afin, ensuite, de souligner les stratégies qu'elles ont mis en œuvre et de les confronter à mes hypothèses de départ.

\section{Repère historiographique et méthodologie}

4 Cette recherche s'inscrit dans la lignée des travaux menés sur la thématique désormais visible et académiquement légitime "genre et migrations». Ainsi, le colloque organisé par MIGRINTER en juin 2016 « Penser les migrations pour repenser la société » dressait un bilan et ouvrait des perspectives en termes d'intersectionnalité. Si cette contribution ne cherche pas à mettre en lumière les formes de discrimination ou de domination dans la société, elle se rapproche néanmoins des travaux de Perla Serfaty-Garzon. Dans ses travaux sur les récits féminins que l'auteure inscrit comme une conquête de soi, la sociologue fait le constat que des migrations de détresse assumées engendrent une grande vitalité (Serfaty-Garzon, 2006). En ce qui concerne la méthodologie, l'analyse repose d'une part sur le concept de stratégies et d'autre part sur trois types de sources, à savoir: des archives familiales, des documents administratifs et des témoignages. Le rapport avec ces deux derniers types de source nécessite d'être explicité.

\subsection{Le témoignage}

D'entrée, le témoignage n'est pas appelé à la barre pour dire $L a$ véritét. D'abord, parce qu'il n'est pas le présent mais la parole de maintenant sur ce qui s'est passé. Ensuite, parce que l'historien'ne à travers le témoignage ne cherche pas la vérité des faits mais une expérience. Ainsi, si pour François Maspero le témoignage peut parfois se révéler en partie faillible, le propos n'est pas d'apporter un témoignage qui aurait la précision d'un procès-verbal, mais de transmettre quelque chose, même infime, dans son cas, « de la vie du camp et de son odeur de mort [...] cela compense mille fois telle ou telle imprécision factuelle $^{7} »$ (Maspero, $2002: 14$ ). Le témoignage est donc pris comme une source et ne doit 
pas faire craindre à l'historien-ne le recueil d'anecdotes, pas plus que la contingence. Pour ce faire, ilelle doit se « laisser travailler par son matériau ", se mettre à l'écoute, « laisser flotter son attention" (Pétonnet, 1982: 37-47). Il-elle ne doit pas s'enfermer dans ses hypothèses et ses données, mais adopter un principe de réflexivité : «[...] se départir de ses idées reçues, de ses prénotions et de ses préconceptions. Non pas s'en défaire [...] mais les neutraliser. » La réflexivité est un sens de l'imagination qui s'autolimite, elle est aussi "méfiance incorporée vis-à-vis de toutes [...] idéologies ", méfiance face à un excès de certitude, pour un doute permanent (Céfaï, $2003: 524$ ). Le parti pris est donc celui de laisser dire, de laisser surgir et de tourner son attention vers un passé. Cependant, quelle que soit la posture adoptée par l'historienne, une tension peut se créer si le rôle de chacune n'a pas été clairement explicité. C'est là toute la question du statut du témoin : acteur ou informateur? Car si le témoin a le choix de concevoir son témoignage comme un devoir de mémoire il ne saurait «négliger l'exigence de vérité qui est au cœur du travail de l'historien-ne. C'est à cette [...] condition que la mémoire sociale pourra faire son travail de rattachement au passé, en évitant la mythologie sans tomber dans l'oubli » (Vernant, 2004 : 131-132). Énoncer ces écueils pourrait paraître vain; pourtant, savoir qu'ils existent devrait aider à les éviter.

\subsection{Les sources écrites}

6 Les Archives départementales du Rhône conservent plusieurs dizaines de milliers de dossiers d'étrangers sous la côte $829 \mathrm{~W}$. Le mémento rédigé à l'intention des fonctionnaires, maires ou employés de mairie présente les attributions du $3^{\mathrm{e}}$ bureau de la

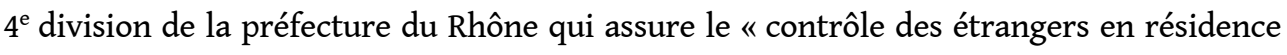
dans le Rhône $»^{8}$. Le sommaire suffira ici: il s'agit notamment de procéder à la "délivrance et au renouvellement des cartes d'identité », à la rédaction de «toute la correspondance générale concernant le contrôle des étrangers ». Chacun des dossiers d'étrangers, numéroté, est un voyage incertain vers le vivant, l'épaisseur d'une vie, la chair d'un être. Chaque dossier du $829 \mathrm{~W}$ entraîne un oubli de soi, une fusion avec cet être dont on espère qu'il est parvenu à un équilibre de vie dans sa situation d'étrangerère pendant et au sortir de la guerre. C'est par le biais de ces dossiers d'étrangers qu'il m'a été donné de rencontrer Aldona Starzynska et Irena Borsukowska.

7 Ce matériau de travail, fait de documents familiaux, de procédures et d'imprimés administratifs et de témoignages sont exploités autour d'un questionnement relevant du concept de stratégie.

\subsection{Stratégies}

Selon Hegel, le pouvoir de domination est le fruit d'une auto-domination portée par le'la dominée et n'existerait que parce qu'internalisé comme une détermination permanente. Adhérer à cette vision sans la questionner revient à nier la notion de conscience individuelle, mais aussi la capacité de chacune à remettre en question cette détermination pensée comme permanente. Or, les hommes et les femmes sont douée's d'une conscience qui, par définition, fait d'elles·eux des acteurstrices. Ilselles se déterminent tous'tes en fonction de leur degré de conscience au monde. S'affranchir de cette détermination permanente, c'est mettre en œuvre des stratégies de rapport au monde, au pouvoir, au dominant. Chaque parcours de vie est le résultat de choix et 
d'absence de choix, de décisions et de non-décisions, où le destin et le hasard côtoient l'arbitraire du pouvoir et la fatalité, mais où l'individu occupe une place centrale et est doté de pouvoirs d'action plus ou moins importants selon le contexte, le genre, l'âge, le statut social, matrimonial, professionnel, etc. C'est en cela qu'il existe des stratégies. Ainsi, ces éléments méthodologiques permettent de poser la question : depuis la place que la société hiérarchisée cherche à leur imposer, que peuvent produire, induire, construire ces femmes pour infléchir, orienter leur parcours de vie, en réaction à la situation dans laquelle elles sont placées.

\section{Parcours de vie}

\subsection{Aldona Starzynska9}

Le 13 mars 1944, Wanda Starzynska voit le jour à l'Hôtel-Dieu, au cœur de la ville de Lyon. Sa mère, Aldona, vient de donner naissance à son second enfant. Mais quels sont les sentiments qui dominent? Joie ou détresse ? Courage ou abattement? Son mari Andrzej Starzynski n'est pas là pour accueillir l'enfant. Il est absent depuis plusieurs mois, exactement depuis le 20 septembre 1943. Ce jour-là, Andrzej Starzynski n'est pas revenu à leur domicile après avoir achevé sa journée de travail au bureau d'administration des Polonais en France ${ }^{10}$. Mais peut-on encore parler de travail ? Depuis le $1^{\text {er }}$ septembre 1939, la Pologne a été envahie, démembrée et rayée de la carte de l'Europe. Des dizaines de milliers de Polonais ont poursuivi le combat depuis la France au sein d'une armée polonaise reconstituée et placée sous le commandement du gouvernement polonais en exil à Londres. Lorsque le maréchal Pétain, de sa voix chevrotante, a déclaré le 17 juin 1940 qu'il «faut cesser le combat », ces mêmes hommes, à l'appel de leur gouvernement, ont poursuivi la lutte et initié des réseaux de renseignements. Andrzej Starzynski est de ceux-là. Né en 1905 à Zezulin dans le voïvode de Leczna près de Lublin, il est issu de la petite noblesse. Dès l'âge de 20 ans, Andrzej décide de quitter ses parents ainsi que ses six frères et sœurs pour rejoindre la France et y poursuivre des études de droit. Embauché par le consulat de Lille à une date incertaine, il y fait connaissance d'une secrétaire, recrutée quelques temps après, Aldona Mikulski. Petite femme mince aux cheveux noirs, elle est née en 1904 à Stawiszcze (Stavyshche) en Ukraine, près de Kiev, et est également de nationalité polonaise. Issue d'une famille ennoblie au XVII ${ }^{\mathrm{e}}$ siècle, son enfance est riche d'enseignements: celui d'une gouvernante française, qui lui apprend la langue de Molière, mais aussi de l'ouverture d'esprit d'une mère artiste peintre et d'un père pharmacien et juge de paix. La famille reçoit beaucoup d'ami-e's, des intellectuels, artistes notamment. La Révolution russe sonne le glas de ces années d'insouciance. Leur maison est au cœur des combats, tantôt occupée par les soldats de l'Armée blanche, tantôt par les bolchéviques ${ }^{11}$. À la mort de son père, Aldona s'enfuit avec ses grand-mère, mère et sœur. Quatre femmes au cœur de la tourmente de la guerre civile emportent ce qu'elles peuvent et cherchent, en ce mois d'août 1921, à gagner Varsovie. Elles y parviennent pendant l'hiver 1921-1922, mais trois décèdent, les 11 et 16 janvier 1922, du typhus et d'une pneumonie. Aldona reste seule, mais réussit à retrouver une grande tante restée à Varsovie. Elle y dépose les reliques d'une histoire familiale sous forme de dessins, d'objets, de documents officiels et de bijoux. Ces derniers seront vendus pour la plupart au fur et à mesure des nécessités vitales qu'impose la condition humaine : manger, s'habiller, se chauffer. Pourtant, Aldona reprend sa scolarité, commence des études, mais 
la tuberculose l'oblige à s'arrêter et se soigner dans le sud du pays, à Zakopane. À sa guérison, elle décide de ne pas poursuivre et, grâce à sa connaissance du français, trouve un emploi de secrétaire au consulat de Lille où elle arrive en 1927-1928. Des photos la montrent en compagnie d'amis et de son futur mari. Une jeune femme espiègle, qui a de l'esprit, qui s'amuse, vit. C'est en 1935 que les jeunes gens se marient, à Varsovie, où ils restent quelques mois avant d'effectuer un voyage de noce à Venise et de reprendre leurs postes au consulat de Lille du $1^{\mathrm{er}}$ mars au 30 septembre 1936 puis d'être mutés à Lyon le 1 er octobre. C'est là que la guerre les saisit. Andrzej participe le 20 octobre 1939 à la commission chargée du recensement « de tout citoyen polonais du sexe masculin résidant ou de passage en France n'ayant pas souscrit d'engagement volontaire dans les rangs de l'armée française ${ }^{12} »$. On peut supposer qu'il se rend ensuite à Parthenay où une armée polonaise en France est en cours de constitution. De là, il est affecté au centre d'instruction DCA de Saint-Nazaire d'où il adresse des lettres à Aldona. Son dossier de résistant ${ }^{13}$ atteste de sa participation active à la guerre de 1940 . On le retrouve ensuite à Lyon où, après la défaite, il a repris ses fonctions au sein de l'administration polonaise en France. Il est attesté qu'à partir du $1^{\text {er }}$ décembre 1942 il travaille officiellement pour la Résistance polonaise en France au sein du réseau F2 de la POWN ${ }^{14}$. Le 20 septembre 1943, il est arrêté par la Gestapo dans les locaux de cette administration et emmené à la prison allemande de Montluc (Lyon) ${ }^{15}$. Il y est torturé avant d'être déporté le 19 janvier 1944 vers le camp de concentration de Buchenwald ${ }^{16}$.

\subsection{Irena Borskukowska ${ }^{17}$}

Irena Borsukowska voit le jour le 6 mai 1917 à Jekaterynoslaw, aujourd'hui en Ukraine, mais antérieurement en territoire polonais alors annexé par la Russie à la suite du troisième partage de la Pologne en 1795 (ADR, 829 W 457, n ${ }^{\circ}$ 92544). Son père, Alexandre Borsukowski, né en 1886 à Lublin, a étudié à l'école polytechnique de Saint-Pétersbourg avant d'occuper différents postes dans la Russie tsariste ${ }^{18}$. Sa mère, Casimiera Borsukowska née Rotkel, est issue d'une famille polonaise d'ascendance suédoise (ADR, $829 \mathrm{~W} 457, \mathrm{n}^{\circ}$ 92544). Quelques mois après la naissance d'Irena, la famille est contrainte de tout quitter afin d'échapper au déchaînement dévastateur de la Révolution russe et rejoint la Pologne. C'est, dès sa toute petite enfance, le premier déracinement d'une longue série. Cependant, dès 1918, après cent cinquante ans d'inexistence, l'État polonais renaît de ses cendres. La famille réside d'abord dans la région de Lublin et lorsqu'Alexandre Borsukowski est appelé à Varsovie à une date incertain $\mathrm{e}^{19}$ pour assumer des fonctions officielles dans le nouveau gouvernement, elle s'installe dans la capitale polonaise. Pour Irena commencent des années d'insouciance et ses enfants soulignent que leur mère "a sans aucun doute vécu une jeunesse heureuse dans un milieu social confortable ${ }^{20} »$. Elle étudie, apprend le français à l'école et en 1939 commence des études supérieures. C'est là qu'elle assiste probablement au bombardement de Varsovie.

On allait vers l'automne, mais il faisait encore très chaud. Depuis des années, nous n'avions pas eu un été aussi beau. [...] le 31 août, la soirée était splendide. [...] nous nous sommes couchés tard. [...] Nous dormions fenêtres ouvertes. À cinq heures du matin, nous fûmes réveillés par de fortes explosions. Je me précipitai vers la fenêtre. Le ciel était rouge sang. (Reicher, 1996 : 13-14)

Pendant trois semaines, la Pologne résiste avant d'être vaincue et de nouveau démantelée entre l'URSS et l'Allemagne. Il est indéniable que dans la mesure où Alexandre Borsukowski appartient à l'intelligentsia polonaise, sa vie est en danger. Du côté de sa 
femme, la moitié des hommes sont assassinés dans les forêts de Katyn par l'Armée rouge ${ }^{21}$ . La guerre interrompt les études d'Irena, les universités sont fermées et, de 1940 à 1944, la famille doit déménager après que leur appartement a été détruit dans un bombardement et va s'installer dans le « petit ghetto » juif après que celui-ci a été liquidé, c'est-à-dire que les Juif·ve's ont été déportée's. Lorsqu'éclate l'insurrection de Varsovie le $1^{\text {er }}$ août $1944^{22}$, ils sont pris au piège et assistent à « l'anéantissement de leur ville » (ADR, $829 \mathrm{~W} 457, \mathrm{n}^{\circ}$ 92544) $)^{23}$. Logeant dans une cave, Irena et sa mère ne sortent pas durant plusieurs semaines; seul le père s'extirpe des décombres de la ville à la nuit tombée pour essayer de trouver de quoi manger. Aucun document consulté, pas plus que les témoignages de la famille, ne permettent d'affirmer qu'ils aient pris part à cette insurrection, mais on sait qu'ils ont été tous trois "déportés de Varsovie lors du soulèvement contre l'Allemagne nazie en 1944 " (ADR, $829 \mathrm{~W} 457, \mathrm{n}^{\circ}$ 92544). Les Allemands mettent soixante-trois jours à vaincre et écraser l'insurrection, avant de vider Varsovie de sa population, puis de raser une grande partie de la ville. Cependant, au fur et à mesure de l'avancée de l'armée allemande, les populations civiles sont déportées vers l'Allemagne. C'est, pour Irena et ses parents, un nouveau déracinement dans la souffrance, un trajet qui s'éternise sur deux interminables semaines au cours desquelles ils parviennent à survivre alors que le nombre de morts ne cesse d'augmenter. La date du $1^{\text {er }}$ septembre 1944 comme jour d'arrivée au camp de concentration de Dachau n'est pas certaine, mais elle est mentionnée dans une enquête de police postérieure aux événements ${ }^{24}$. Irena et sa mère sont séparées de leur père et mari. La chronologie des événements demeure obscure, mais le récit familial rappelle que grâce aux compétences linguistiques d'Alexandre Borsukowski, qui parle couramment sept langues, ses services comme interprète sont requis par l'administration du camp de concentration. Il accepte à condition que sa femme et sa fille soient protégées. Ce même récit familial rapporte qu'alors qu'elles sont déjà dénudées avant d'être poussées vers les chambres à gaz, Irena et sa mère sont appelées et se voient ordonnées de se rhabiller avant de sortir. Jusqu'au 29 avril 1945, date de la libération du camp par les troupes américaines, tous trois réussissent à survivre, malgré le terrible froid de cet hiver-là, malgré la désorganisation et l'aggravation dramatique des conditions de vie dues à l'arrivée de prisonnierère's transférée's des camps proches du front et que les Allemands ne veulent pas libérer, malgré le typhus. Pourtant, le 26 avril, lorsque les Allemands quittent le camp et forcent 7000 prisonniers à se rendre à pied à Tegernsee plus au sud, Alexandre, sa femme Casimiera et Irena n'en sont pas. Se sont-ils cachés ? Ont-ils obtenu de l'aide à l'intérieur du camp du fait de contacts d'Alexandre Borsukowski avec l'administration du camp ? Ou, parce qu'ils ne sont pas Juif·ve's, ne sont-ils pas entraînés dans ces marches de la mort ?

Il s'agit à présent de s'interroger sur les stratégies de survie et de vie mises en œuvre par des deux jeunes femmes. L'horreur de la déportation et les stratégies de survie ne sont pas étudiées ici, je m'intéresserai davantage à la survie, au sortir de la guerre pour Irena, jusqu'à la Libération pour Aldona, avant d'analyser les liens qu'elles ont tissées après leur rencontre à Lyon. 


\section{Stratégies de survie}

\subsection{Aldona Starzynska}

Le 20 septembre 1943, tous les ancien'ne's agente-s diplomatiques et consulaires polonais du bureau lyonnais d'administration des Polonais en France et de l'Union des Polonais en France sont arrêtés par la Gestapo ${ }^{25}$. À cette époque, le bureau est dirigé par le ministre plénipotentiaire français Ch. Billecocq assisté de Paul Duvivier qui interviennent en faveur du personnel incarcéré. Le premier s'adresse à l'intendance de police dès le 23 septembre afin d'obtenir que mesdames Mrozkiewicz et Szpakowska soient libérées, ainsi que le garçon de bureau Feliks Zmyslony. Il ne demande pas la libération d'Andrzej Starzynski et de Franciszek Karecki qui sont accusés « d'agissements anti-allemands ». Il réclame pour eux la possibilité que leur famille apporte à Montluc «des effets d'habillement et de toilette ${ }^{26}$ ». Le 24, alors que personne n'a été relâché, Paul Duvivier intervient à son tour. Est-ce le résultat de cette intervention qui conduit à la libération de tous, à l'exception d'Andrzej Starzynski? On ne peut que supputer qu'Aldona comprend la gravité de la situation, bien que, selon sa fille, elle n'était peut-être pas au courant de l'implication de son mari dans la Résistance polonaise. Selon un témoignage postérieur de Marie Mrozkiewic ${ }^{27}$, les agents de la Gestapo perquisitionnent chez Aldona et reviennent à plusieurs reprises pour l'interroger au sujet des activités de son mari, la menaçant et lui interdisant de quitter Lyon $^{28}$. Le danger est réel puisque d'autres épouses ont été arrêtées en octobre et déportées ${ }^{29}$. Aussi, Aldona quitte précipitamment Lyon avec la petite Ada qui a tout juste deux ans. Aldona sait-elle qu'elle est enceinte de son deuxième enfant? Andrzej est alors toujours interné à Montluc et pourtant, par stratégie de survie, elle se réfugie avec Ada à Mondragon dans le Vaucluse chez les Brecieux, des connaissances du frère d'Andrzej, Sigmund Starzynski, lui-même réfugié de Pologne en 1940 puis parti se battre depuis l'Angleterre. Il y a bien ici la mise en service d'un réseau d'entraide, à la fois familial et professionnel sur lequel elle peut compter. En effet, c'est avec l'aide de Marie Mrozkiewicz qu'elle prépare d'urgence ce départ et c'est assurément grâce à la Résistance polonaise qu'elle monte à bord d'un camion qui se rend dans ce village. Pour une raison non connue mais qui ne peut relever que de sa sécurité, Aldona ne reste pas chez les Brecieux, mais est cachée avec sa petite fille dans une cabane aux alentours du village. Lorsqu'elle revient à Lyon en mars 1944 pour accoucher, Marie Mrozkiewicz a "pu constater son déplorable état de santé, dû au manque de nouvelles de son mari, aux conditions extrêmement dures dans lesquelles elle a passé l'hiver et à la frayeur dans laquelle elle vivait depuis des mois ${ }^{30} »$. Après la naissance de Wanda, Aldona continue de se cacher car la Gestapo s'intéresse toujours aux membres de famille des personnes déportées en Allemagne, d'autant qu'un vaste coup de filet est organisé par la Gestapo au mois de juillet 1944. Au cours de cette même période, les intenses bombardements alliés reprennent dès fin mars 1944 et s'égrènent jusque mi-août. Celui du 26 mai 1944 est effectué par l'aviation américaine et vise les installations ferroviaires. Or, leur appartement sis au 35 rue Général Miribel donne directement sur les voies ferrées qui conduisent à la gare de marchandises de Lyon-Guillotière. Ce matin-là, elles se réfugient dans un abri anti-aérien, au $\mathrm{n}^{\circ} 7$ de leur rue, mais ce dernier ne résiste pas et s'effondre. Il $\mathrm{y}$ a des morts mais toutes trois en réchappent, ce qui vaudra à Wanda d'être appelée par une voisine «la miraculée ${ }^{31}$. À une autre occasion, Aldona se promène au parc de la Tête-d'Or, au nord de la ville, lorsque les sirènes retentissent. Elle trouve abri dans un 
bâtiment scolaire réquisitionné par les Allemands pour en faire un hôpital militaire ${ }^{32}$. Alors qu'elle prend conscience du lieu où elle se trouve et veut s'enfuir, une sentinelle allemande la retient. À ses filles, elle racontera plus tard ne pas avoir su de quoi elle avait eu le plus peur : de l'alerte ou de s'être réfugiée dans ce que la mémoire familiale retient comme la Kommandantur. Ce glissement mémoriel d'un lieu de soin à un lieu de souffrance associé à l'arrestation, la torture et la déportation de son mari, éclaire l'ampleur du traumatisme vécu par Aldona ${ }^{33}$.

Après la Libération de Lyon le 2 septembre 1944, on peut penser qu'Aldona retourne définitivement chez elle avec ses deux filles et attend son mari tout en multipliant les démarches. Au cours de sa déportation, Andrzej parvient à adresser plusieurs cartes postales à sa femme, entre le 27 février 1944 et le 18 juin 1944. Puis, plus rien, hormis le silence assourdissant de l'attente. C'est un télégramme qui le rompt : elle reçoit l'annonce de la mort de son mari, exécuté le 21 mars 1945 par pendaison au camp de Mittelbau-Dora où il avait été transféré le jour de la naissance de sa fille un an auparavant. Désormais, Aldona reste seule avec ses deux filles qui sont, comme elle le dira elle-même, sa source de motivation.

\subsection{Irena Borsukowska}

Lors de la libération des camps, tous les ancien-ne's déportée's sont pris·es en charge par la United Nations Relief and Rehabilitation Administration (UNRRA), créée le 9 novembre 1943 par les troupes alliées. L'un des enjeux de l'UNRRA est de rendre à leur vie d'avantguerre des millions d'hommes et de femmes déplacée's et regroupée's sous le terme générique de Displaced Persons (DPs). Pour cela, les DPs sont placée'es dans des camps dont ils et elles n'ont pas la gestion, pas plus qu'ils et elles n'ont la possibilité de travailler et donc de commencer à se reconstruire une identité conforme à celle qui était la leur avant-guerre. En ce qui concerne la famille Borsukowski, que signifierait un retour à une vie « normale » en Pologne? Leur famille a été décimée par la guerre, leur logement a été détruit lors de l'écrasement de l'insurrection de Varsovie et il ne fait aucun doute que les contacts du chef de famille avec le gouvernement polonais en exil à Londres ne seraient pas appréciés des nouvelles autorités en place, à savoir le gouvernement provisoire de la République de Pologne, d'obédience communiste et à la solde de l'URSS ${ }^{34}$. En d'autres termes, un retour en Pologne n'est pas envisageable.

La chronique familiale retient qu'Alexandre Borsukowski est à l'origine de la stratégie de survie adoptée par la famille: s'exiler en France, ce qui constitue un troisième déracinement. Lors de la conférence de Yalta de février 1945, il a été décidé par les commandements alliés que le rapatriement se ferait selon un schéma qu'un témoin résume ainsi: "Les Occidentaux vers l'Ouest, les Slaves de l'autre côté." (Evrard, 1972 : 433) Cela signifie que ne peuvent être rapatriée's en France que celles et ceux qui y vivaient avant le $1^{\mathrm{er}}$ septembre 1939, à moins qu'il ne s'agisse de l'épouse étrangère d'un déporté du travail ou d'un prisonnier de guerre en Allemagne ${ }^{35}$. La chronologie exacte n'est pas connue. À quel moment Irena Borsukowska fait-elle la connaissance de François Privey ? Jeune Lyonnais d'une vingtaine d'années, il a été déporté après avoir été dénoncé par un camarade de classe entré dans la Milice : requis du STO, il avait refusé de partir et s'était réfugié dans le Vercors. C'est au cours d'une visite à Lyon qu'il est arrêté puis déporté en Allemagne. Selon une enquête de 1946 à laquelle il a répondu, François Privey a travaillé pour la compagnie des chemins de fer allemands à Munich à partir du 16 juin 
1943. Selon son fils Marc, François Privey était entre autres chargé de l'entretien du camp de concentration de Dachau alors que lui-même se trouvait dans un camp de travail ${ }^{36}$. Cependant, ce qui est certain, est que son chemin croise celui d'Alexandre Borsukowski. Ce dernier ne manque pas de remarquer la haute stature du jeune homme, une personnalité droite et solide. Marc Privey affirme qu'il y a eu une confiance et une estime réciproques entre les hommes. C'est lui qui le présente à sa fille ${ }^{37}$. Ici encore la chronologie est incertaine, mais cela se passe avant la libération du camp de Dachau le 29 avril 1945. On peut penser qu'après le départ des SS le 26 avril, François Privey s'entend avec Alexandre Borsukowski pour fuir vers l'Ouest afin de ne pas être libérée's par les Soviétiques. C'est donc lorsqu'elle s'échappe du camp de concentration avec sa mère qu'Irena fait la connaissance de François Privey. Un mois plus tard, le 31 mai 1945 les jeunes gens se marient en Allemagne, formalité indispensable pour leur entrée en France le 4 juin 1945. On est en droit de se demander dans quelle mesure Irena a fait le choix d'épouser ce cheminot ${ }^{38}$. Il est avéré que le père exerce une influence très forte sur la fille en même temps que cette dernière est douée d'une volonté de fer et a bénéficié d'une éducation moderne et émancipatrice. Aussi, pour expliquer la personnalité de sa mère, Élisabeth Privey parle d'adéquation entre le père et la fille, d'une adhésion de celleci à l'analyse de son père : elle sait le retour en Pologne impossible, elle sait l'estime de son père pour le jeune Français, elle comprend qu'il est un homme sur lequel elle pourra compter. Cette union apparaît d'autant plus comme une stratégie que François Privey est déjà promis à une autre en France et on comprend d'un homme dont la droiture ne cesse d'être invoquée dans les entretiens qu'il n'a pu qu'être séduit par la personnalité du père ou par cette jeune fille amaigrie et affaiblie par des années de privations. Cependant, il est possible de formuler l'hypothèse d'un lien social créé en dehors des structures conventionnelles et que l'historien Thomas Kühne (2006) nomme « social bonding ». Dans ses travaux sur les situations sociétales comme les camps de travail, les camps de réfugiée's, il a montré que les hommes et les femmes cherchent inconsciemment à recréer des cadres relationnels pour tenter de s'intégrer dans une certaine conformité, de s'impliquer dans des activités conventionnelles dont ils croient en la valeur morale, ou plus simplement pour marquer un attachement aux autres. De plus, après presque deux ans de déportation du travail, de qui François Privey se sent-il plus proche ? De cette famille qu'il a appris à connaître et estimer, ou d'une promise qu'il connaît peut-être à peine? Pour Élisabeth Privey, sa mère a choisi sous la contrainte du moment et elle ajoute: «[...] la guerre, quand vous êtes dans une situation désespérée, vous faites le moins mauvais choix ${ }^{39}$. » Néanmoins, pour Élisabeth Privey comme pour son frère Marc, leurs parents se sont choisis, ont été très unis et ont construit un foyer à l'atmosphère détendue et aimante.

\section{Stratégies de vie}

17 Lorsqu'Irena arrive à Lyon au bras de son mari de cinq ans son cadet, le premier accueil de sa belle-famille n'est pas des plus chaleureux, ce qui n'est pas un cas isolé. Comme le fait remarquer l'abbé Glasberg, acteur majeur de la période dans la protection des réfugiée'es,

Beaucoup d'hommes, [...] cèdent à l'indignation de la famille dressée contre l'intruse. Parfois cette famille a cruellement souffert, à l'insu du fils ou du frère revenu au loin, et sa rancune est compréhensible. Mais parfois aussi, elle ne fait que céder au préjugé, à la simple haine contre l'étrangère. (Glasberg, 1946 : 49-51) 
De plus, ce fils était déjà promis. Néanmoins, Irena est rapidement acceptée par la famille Privey et après quelques mois de vie chez ses beaux-parents, impasse colonel Lamy, le jeune couple emménage dès septembre 1945 dans ce que leur fils qualifie de taudis, 43 avenue Viviani dans le $8^{\mathrm{e}}$ arrondissement, à la limite de la ville, presque un terrain vague ou déjà la campagne. $25 \mathrm{~m}^{2}$ insalubres où les rejoignent les parents d'Irena le 12 avril 1946 et un bébé, Élisabeth, née le 30 mai 1946.

De son côté, Aldona Starzynska vit avec ses deux filles dans leur appartement du $7^{\mathrm{e}}$ arrondissement, travaille un temps au nouveau consulat, puis y renonce lorsqu'on lui demande d'espionner pour le compte des nouvelles autorités polonaises. Le cercle de compatriotes anciennement fonctionnaires du consulat s'étiole nécessairement avec l'orientation politique des nouvelles autorités polonaises.

20 À la question de savoir comment leurs mères se sont rencontrées alors qu'elles ne vivent pas dans le même quartier et que treize ans les séparent, les enfants Privey suggèrent que c'est par l'intermédiaire des maisons familiales liées à l'Église catholique, tandis que Wanda Starzynska évoque le Dom Polski, une association qui existait avant-guerre et maintenait le lien entre les ressortissantees de culture polonaise.

\section{Qu'est-ce qui lie ces femmes?}

21 À la lecture des dossiers administratifs qui donnent à construire une certaine image de ces deux femmes, l'hypothèse de départ pour expliquer leur rapprochement est leur origine sociale commune. Non seulement elles appartiennent à l'intelligentsia polonaise, mais sont des réfugiées du nouveau régime en place en Pologne, sans compter que toutes deux ont appris le français du fait de leur éducation. Les témoignages des enfants ont permis de discuter cette hypothèse.

\subsection{Des personnalités fortes}

Les enfants rapportent tous que leurs mères avaient une forte personnalité. Pour Élisabeth, au sein du couple, Irena avait le dernier mot, même si en apparence, du fait de son éducation, elle se plaçait sous l'autorité de son mari. Ce qui ne l'empêche pas d'avoir une volonté de fer et une indépendance d'esprit. Wanda donne de sa mère Aldona une image plus contrastée, celle d'une petite femme en robe noire et meurtrie par le veuvage, nerveuse, en même temps qu'une femme de caractère, sympathique et séduisante. Par ailleurs, Marc Privey souligne que de nombreux moments de bonheur familiaux dans une famille peu démonstrative sont liés à l'humour. Irena et ses parents le pratiquent avec élégance et se livrent à l'autodérision sur les soucis de la vie quotidienne qui n'ont rien de commun avec les drames de leur vie pour lesquels à l'évidence ils n'y ont pas recours. Wanda rapporte que sa mère Aldona aimait rire, jouer avec les mots, tout en " ayant du culot et de la répartie ». Elle se remémore son sens de l'humour et une forme d'autodérision: «Quand elle voyait des choses qui pouvaient indigner, elle essayait d'en rire, elle avait une belle réaction puis elle disait que c'était pittoresque ${ }^{40}$. " Enfin, comment ces femmes auraient-elles pu survivre à ces parcours chaotiques si elles n'avaient pas été douées d'une forte personnalité? 


\subsection{Une communauté de parcours}

23 L'hypothèse formulée était qu'Aldona et Irena avaient développé une relation forte. Les entretiens menés n'ont pas d'emblée confirmé cette hypothèse, mais des éléments ténus ont néanmoins pesé en faveur de cette représentation. Lorsque Wanda réfléchit à son enfance, elle ne considère pas de prime abord que des liens privilégiés aient uni leurs familles. Pourtant, elle se remémore ensuite qu'elle a visité les Borsukowski, entre autres familles ${ }^{41}$. Elle décrit leur maison comme « une baraque pas possible en bois. À l'intérieur, il y a une pièce vraiment très étroite, [...] on est serré autour de la table et la grand-mère nous fait des tartines avec un demi-centimètre de beurre et du miel par-dessus ». Des photos prises dans le terrain attenant montrent les trois filles en compagnie d'Irena. Puis, de sa mémoire surgit une anecdote à propos de Marc Privey : «Marc, je l'ai vu dans son landau et on me disait, ce sera ton fiancé (rires). Moi j'avais au moins dix ans et je n'avais pas l'intention d'épouser quelqu'un qui a dix ans de moins que moi ${ }^{42}$. " Ces éléments ténus permettent d'affirmer qu'entre les mères des liens forts se sont tissés, que les enfants ont ressenti et conservé dans leur inconscient, mais qu'ils ont aujourd'hui du mal à définir clairement. Pour autant, en ce qui concerne l'appartenance à une même classe sociale ou à une même nationalité comme facteurs de rapprochement, Élisabeth ne les considère pas comme primordiaux. Certes, les deux femmes partagent une même éducation, une même culture, parlent ensemble le polonais, leurs familles sont originaires de Lublin, mais Élisabeth affirme de sa mère que plus que tout, elle avait «tendance à se sentir plus en adéquation avec des gens déracinés ${ }^{43}$ ». Elles sont des déracinées, des réfugiées a priori sans espoir de retour en Pologne. Cette dimension est d'ailleurs profondément ancrée dans la personnalité d'Irena. Son fils Marc se remémore qu'à son départ pour les États-Unis à la fin des années 1970, sa mère s'est tue mais a pensé qu'elle ne reverrait jamais son fils. Dans la mesure où son parcours de vie est jalonné d'une succession de déracinements, depuis l'effondrement de la Russie tsariste, la déportation de Pologne et l'impossible retour après-guerre, sa représentation mentale du départ ne laisse pas de place possible au retour, il n'y a pas d'alternative au déracinement et à l'exil. Élisabeth ajoute que les traumatismes qui ont succédé à une jeunesse dorée et insouciante constituent assurément un facteur de rapprochement entre les mères, lorsque Marc évoque, lui, des éléments de souffrance communs pour l'expliquer. Néanmoins, il ressort de ces entretiens un même profond désir de protéger les enfants. Lorsqu'elle arrive en France, Irena est une jeune femme physiquement épuisée des suites des souffrances endurées, sa santé reste fragile et après la naissance de sa fille en 1946, on lui annonce qu'elle ne survivrait pas à une deuxième grossesse, et on ne lui donne pas dix ans de vie. La naissance de Marc en 1955 est assurément une double victoire de la vie, non seulement parce que l'impensable est arrivé mais que l'état de grossesse a provoqué une régénérescence partielle des cellules. À l'opposé, Wanda est née dans un contexte traumatique de désespérance, mais Aldona s'est également tournée vers la vie et ses filles. Si Irena n'est "pas portée sur les bébés ", laissant cette tâche davantage à sa mère qui aime à s'occuper de la sphère familiale, elle cherche ce qu'il y a de mieux pour ses enfants et soutient sa fille Élisabeth contre l'avis des beaux-parents lorsqu'il s'agit de la laisser poursuivre ses études au Lycée. Marc Privey conclut que leur mère a reproduit avec eux " cette protection qu'elle avait vécu avec ses parents ». Pour Wanda, sa mère est « maman Noël ». Elle les protège, se consacre à elles. Pour cela, elle construit une ossature de vie où les enfants occupent la place centrale, ce qu'elles ressentent car elles ne supportent pas 
l'intrusion d'un homme auprès de leur mère : «C'était notre mère et elle devait rester notre mère. » Les souvenirs de Wanda sont émaillés de moments heureux, notamment la profusion de cadeaux à Noël, les sorties hebdomadaires à la bibliothèque et au salon de thé, etc. Elle parle « d'enfance magique ». De même, Aldona soutient ses filles dans leurs projets d'études, cherche toutes les solutions pour soigner Wanda qui souffre d'asthme aigu qui nécessite des soins et des séjours à la campagne ou en cure.

\subsection{Ce qui les différencie}

Pourtant, les deux femmes se différencient sur un certain nombre de points. Chez Wanda, on parle polonais, ce que sa sœur Ada et elle répugnent à faire dans la rue, provoquant ce sentiment de honte connu chez les enfants de migrantees, lorsque le parent parle sa langue d'origine dans un lieu public. Au contraire, dans la famille de Marc et Élisabeth, il est décidé de parler français, du moins en présence du gendre et chef de famille, François, ou quand l'épouse d'Alexandre Borsukowski ne comprend pas la conversation du fait de sa connaissance plus limitée du français. Par ailleurs, Irena est devenue Irène, a pris la nationalité française de son mari, tandis qu'Aldona est restée polonaise, réfugiée politique, jusqu'à la fin de ses jours. Paradoxalement, elles n'ont pas manifesté le même attachement aux valeurs traditionnelles polonaises, Aldona s'en écartant davantage qu'Irena. En Pologne, la conception traditionnelle de la place de la femme ne les destine pas à l'exercice du pouvoir, mais à la soumission à l'autorité du mari, à la maison. Lors de la reconstitution de la Pologne en 1917, la répartition des rôles genrés est définie dans la continuité de l'idéologie du combat pour l'indépendance et de la renaissance de la Nation. Les nationalistes polonais encouragent les femmes à suivre l'idée appelée Matka Polka (la mère patriote polonaise), où les femmes sont chargées de l'éducation des enfants dans un esprit patriotique et catholique, du maintien de la langue et des traditions polonaises (Fidelis, 2004). L'État polonais et particulièrement l'Église catholique promeuvent cet idéal maternel dans toutes les couches de la société. Ce modèle qui prédomine parmi les classes aisées a été inculqué à Irena, tandis qu'on est en droit de penser qu'orpheline de père dès son adolescence puis de mère à peine âgée de 17 ans, Aldona n'en a pas bénéficié. En conséquence, Aldona s'est d'une certaine manière affranchie de ces principes, à la fois en termes de domination masculine et d'indépendance financière, ce qu'Irena a partiellement réussi. Dans la mesure où elle est fille unique, elle a toujours joué le rôle du fils et de la fille, a accepté l'autorité de son mari sans pourtant perdre toute marge de manœuvre. Toutefois, malgré sa faible constitution, conséquence des privations dues à la guerre, et à défaut d'une indépendance financière à laquelle elle ne peut accéder, Irena jouit d'une indépendance d'esprit. De plus, à l'image de ce choix de la France pour Irena et du maintien dans la polonité pour Aldona, les réseaux d'intégration à la société française ont été différents et sont le reflet d'un projet d'insertion différent. Les maisons familiales ont été mentionnées pour expliquer la rencontre des mères. Selon Marc Privey, celles-ci ont permis l'insertion d'Irena qui est devenue un membre très actif dans ce cercle, tout en se rapprochant des personnes en situation de déracinement comme elle. Enfin, il est juste de dire que la religion catholique qu'Irena pratique avec ferveur est un élément fort dans la solidité de son couple, de la famille, de son ouverture sur les autres. Quant à Aldona, on peut penser que la religion est un élément de sa culture polonaise, qu'elle transmet mais sans effusion; Wanda rappelle qu'elle ne communiait pas, ce qui peut être lu comme un signe de distanciation vis-à-vis d'une pratique. 


\section{Conclusion}

25 parole. L'émotion ressentie lors de la découverte de leur parcours est entrée en résonnance avec une ego-histoire évoquée en introduction et tenue pour garante d'une analyse critique. Au-delà de l'apparente normalité de ces parcours se dessine l'impérative nécessité de vouloir. Vivre, survivre. Cependant, dans la mesure où les témoignages des enfants sont par définition indirects, ils ne constituent pas des preuves intangibles, mais soutiennent néanmoins un faisceau de présomptions. Il est possible de soutenir qu'à l'origine de la relation entre Aldona et Irena, qu'on ne saurait d'ailleurs qualifier d'amitié, se trouve assurément un commun destin de déracinées, d'exilées, d'étrangères. En conséquence, les liens tissés se révèlent être tout autant l'expression d'une solidarité féminine qu'une solidarité de l'exil, quand une solidarité de classe, si elle n'a pas été formellement retenue, ne peut être totalement écartée. Plutôt qu'à de l'amertume, ce terreau a donné naissance à des parcours de vie en émigration où tout a été fait pour que les enfants puissent se construire et s'enraciner. Par ailleurs, il semblerait qu'une meilleure compréhension des destins en migration devrait s'appuyer sur le concept de social bonding afin de permettre d'interroger sous un angle plus transversal la nature des stratégies tout en questionnant l'intersectionnalité. Ce champ reste à explorer.

\section{BIBLIOGRAPHIE}

BOURDIEU Pierre (1998), La domination masculine, Paris : Seuil.

CAPDeVILA Luc, RouQUeT François, VIRGILI Fabrice \& Voldman Danièle (2003), Hommes et femmes dans la France en Guerre (1914-1945), Paris : Payot.

CÉFAï Daniel (2003), L'enquête de terrain, Paris : La Découverte.

EVRARD Jacques (1972), La déportation des travailleurs français dans le III ${ }^{e}$ Reich, Paris : Fayard.

FARGE Arlette (1997), « Proximités pensables et inégalités flagrantes. Paris, XVIII siècle », C. Dauphin \& A. Farge (dir.), De la violence des femmes, Paris : Albin Michel, 73-87.

FebVRe Lucien (1953), Combats pour l'histoire, Paris : Armand Colin.

GLASBERG Abbé (1946), À la recherche d'une patrie, Paris : Éd. Réalités.

KüHNE Thomas (2006), Kameradschaft. Die Soldaten des nationalsozialistischen Krieges und das 20. Jahrhundert, Göttingen : Vandenhoeck und Ruprecht.

LAPIERRE Nicole (2001), Le silence de la mémoire. À la recherche des Juifs de Płock, Paris : Le livre de poche.

LE FeUVRE Nicky (2004), « Un point de vue sociologique sur les femmes à l'épreuve des années 1940 », J. Fijalkow (dir.), Les femmes dans les années quarante. Juives et non-Juives, souffrances et résistance, Paris : Les Éditions de Paris, 239-244.

ILCEA, 34 | 2019 
FIDELIS Malgorzata (2004), « Equality through Protection: The Politics of Women's Employment in Post War Poland, 1945-1956 », Slavic Review, 63(2), 301-324.

MASPERO François (2002), Les abeilles \& la guêpe, Paris : Seuil.

MUEL-DREYFus Francine, «Le féminin sous Vichy : une catégorie "apolitique” au service de la politique ", J. Fijalkow (dir.), Les femmes dans les années quarante. Juives et non-Juives, souffrances et résistance, Paris : Les Éditions de Paris, 51-62.

PÉTONNET Colette (1982), « L'observation flottante. L'exemple d'un cimetière parisien », L'Homme, $22(4), 37-47$.

PRost Antoine (1966), Douze leçons sur l'histoire, Paris : Seuil.

REICHER Edward (1996), Une vie de juif. L'odyssée d'un médecin juif en Pologne, 1939-1945, Paris :

L'Harmattan.

Sсотт Joan W. (1988), Gender and the Politics of History, New York : Columbia University Press.

ScotT Joan W. (1998), La citoyenne paradoxale : les féministes françaises et les droits de l'Homme, Paris : Albin Michel. (Ouvrage original publié en 1996 sous le titre Only Paradoxes to Offer. French Feminists and the Rights of Man, Harvard : Harvard University Press.)

SERFATY-GARZON Perla (2006), Enfin chez soi ? Récits féminins de vie et de migration, Paris : Bayard.

THEBAUD Françoise (2005), « Genre et histoire en France. Les usages d'un terme et d'une catégorie d'analyse », Hypothèses, 8(1), 267-276.

VERNANT Jean-Pierre (2004), La traversée des frontières, Paris : Seuil.

\section{NOTES}

1. STO : Service du travail obligatoire. La loi du 26 février 1943 rend le travail obligatoire en Allemagne par le biais de la conscription.

2. Je remercie Wanda Starzynska de m'avoir accueillie chez elle, Élisabeth Privey et Marc Privey d'avoir accepté de dialoguer avec moi.

3. L'État polonais renaît de ses cendres à l'issue du traité de Versailles (1918) et du traité de Riga (1921) qui fixe ses frontières.

4. Pierre Bourdieu, Esquisse pour une auto-analyse, Paris, Éditions Raison d'agir, 2004 ; Gérard Noiriel, Penser avec, penser contre. Itinéraire d'un historien, Paris, Belin, 2003. Ils se livrent à cet exercice de manière approfondie. Des historiens dont Michel de Certeau et Antoine Prost ont insisté sur la nécessité de comprendre d'où on parle.

5. Cet article s'appuie sur des recherches menées dans le cadre d'une thèse d'histoire sous la direction de Laurent Douzou et soutenue le 5 décembre 2016: Polonais·es et Juif.ve·s polonais·es réfugiée'es à Lyon (1935-1945). Esquives et stratégies, Université Lyon 2, Lyon, <https://tel.archivesouvertes.fr/tel-01486879/>.

6. Le procès Eichmann qui s'est déroulé en 1961 a marqué l'avènement du témoin. Davantage qu'un verdict, ses organisateurs souhaitaient la constitution d'un récit où le témoignage aurait pour fonction principale, non d'établir la vérité mais de permettre aux victimes de délivrer un message pour la postérité. Voir Annette Wieviorka, L'ère du témoin, Paris, Hachette Littérature, 2002.

7. François Maspero cherche à retrouver des traces de son père mort en déportation. 
8. Archives départementales du Rhône (ADR), 4 M 422. Statistiques des cartes délivrées (1938); états statistiques en vue de la mobilisation (1939) ; états nominatifs des étrangers refoulés par les autorités allemandes de la zone occupée (1940).

9. Les éléments biographiques sont issus des Archives départementales du Rhône (829 W 359, dossier $n^{\circ}$ 76647), de la correspondance entre l'auteure et Wanda Starzynska ainsi que d'entretiens menés en août 2017 à Prignano sulla Secchia (Italie).

10. Ambassades et consulats polonais ont été fermés sur ordre allemand en septembre 1940, remplacés un temps et uniquement en zone libre, par des offices polonais puis par des bureaux d'administration des Polonais en France.

11. Wanda Starzynska conserve des dessins de sa tante qui représentent par exemple le salon dévasté, ou la tombe d'un bolchévique.

12. Archives municipales de Lyon, 1127 WP 004 . Affaires militaires : recensement des Polonais 1932-1945.

13. Service historique de la Défense (SHD), Vincennes, GR 16 P, dossier $n^{\circ} 556250$. Dossiers individuels du bureau Résistance.

14. Il est nommé chef de la Subrégion «Est » le $1^{\text {er }}$ septembre 1943 (Archives familiales Wanda Starzynska, attestation POWN, 12 décembre 1945).

15. ADR, $3335 \mathrm{~W} 4$ et 25 , dossier $\mathrm{n}^{\circ} 000632$. Fichier de la prison de Montluc.

16. Archives familiales Wanda Starzynska. Certificat d'appartenance aux Forces françaises de l'intérieur, réseau POWN-Monica, 5 octobre 1948.

17. Les éléments biographiques sont issus des Archives départementales du Rhône ( 829 W 457, dossier $n^{\circ}$ 92544), de la correspondance entre l'auteure et les enfants d'Irena, Élisabeth et Marc Privey, d'entretiens téléphoniques menés en avril 2014 et mars 2018.

18. La famille a été ennoblie au $\mathrm{XVII}^{\mathrm{e}}$ siècle par Pierre le Grand. Le nom Borsuk est devenu Borsukowski.

19. En toute logique à partir de 1921-1922, c'est-à-dire après que les frontières aient été définitivement tracées à l'issue du traité de Riga du 18 mars 1921.

20. Message de Marc Privey à l'auteure, 19 mars 2018.

21. Au printemps 1940, la police politique de l'Union soviétique massacre plusieurs milliers de Polonais, essentiellement des officiers d'active et de réserve (dont des étudiants, des médecins, des ingénieurs, des enseignants, etc.), et divers autres membres des élites polonaises. La forêt de Katyn dans la région de Smolensk fut le site majeur de ce massacre.

22. Cette insurrection est une conséquence directe de la création du gouvernement de Lublin d'obédience communiste le 22 juillet 1944. L'AK, armée secrète polonaise aux ordres du gouvernement de Londres, décide de soulever Varsovie pour contraindre Moscou à accepter de partager le pouvoir.

23. Lettre de François Privey au préfet, 27 septembre 1945.

24. ADR, 829 W 457, $n^{\circ}$ 92544. Enquête de police du 14 janvier 1946.

25. ADR, $3335 \mathrm{~W} 4$ et 25 , dossier $\mathrm{n}^{\circ} 000632$. Fichier de la prison de Montluc.

26. ADR, $3335 \mathrm{~W} 4$ et 25 , dossier $\mathrm{n}^{\circ} 000632$. Fichier de la prison de Montluc, note manuscrite du directeur du bureau de Lyon, 23 septembre 1943.

27. Marie Mrozkiewicz a participé activement à la Résistance polonaise en France.

28. Archives personnelles Wanda Starzynska. Témoignage tapuscrit de Marie Mrozkiewicz, née Rawska.

29. C'est le cas d'Irène Gradon le 22 octobre 1943 à Lyon (ADR, 3335 W 5, dossier nº 868, fichier de la prison de Montluc). Hélène Malinowski a été arrêtée également le 29 octobre 1943 avec son mari Tadeusz. Incarcérée de longs mois, puis admise à l'hôpital de l'Antiquaille à Lyon, salle des détenues politiques, Hélène Malinowski est conduite le 7 mars 1944 à l'Hôtel-Dieu afin d'y accoucher le 15 mars. Lorsqu'elle y arrive, son état général est déjà très dégradé. Elle décède le 
4 avril des suites de son accouchement (ADR, 3335 W 19, dossier $n^{\circ} 8497$, certificat de l'interne de la maternité de l'Hôtel-Dieu, 25 juillet 1947).

30. Archives personnelles Wanda Starzynska. Témoignage tapuscrit de Marie Mrozkiewicz, née Rawska.

31. Correspondance de l'auteure avec Wanda Starzynska, 3 avril 2018. Sur ce bombardement, voir Bruno Benoit (2017), «Le bombardement de Lyon du 26 mai 1944 et sa récupération politique », en ligne sur Histoires lyonnaises : <https://lyonnais.hypotheses.org/2629> (4 avril 2018).

32. Il s'agit du groupe scolaire au 92-94 rue Tronchet, réquisitionné depuis.

33. Correspondance de l'auteure avec Wanda Starzynska, 3 avril 2018. La Kommandantur était installée dans l'ancienne école de santé militaire, avenue Berthelot.

34. Au cours des six premiers mois de 1945, il existe deux gouvernements polonais qui considèrent chacun être le représentant de la Pologne. Depuis 1940, le Gouvernement polonais en exil à Londres issu de l'intelligentsia polonaise, est la seule instance reconnue par les Alliés. Mais à partir de juillet 1944, l'URSS soutient la constitution du Comité de Lublin d'obédience communiste qui prend le 31 décembre 1944 le titre officiel de Gouvernement provisoire et est finalement reconnu par les Alliés au détriment du gouvernement de Londres.

35. Archives départementales de Savoie, 1398 W 18. Commissariat de police d'Aix-les-Bains au préfet de Savoie, $1^{\text {er }}$ août 1945.

36. L'incertitude du statut de François Privey peut s'expliquer par le fait que la problématique de la détention répression est peu connue : on peut penser qu'à titre répressif, c'est-à-dire pour avoir agi contre le Reich, François Privey a été envoyé en camp de détention à Dachau.

37. Entretien téléphonique avec Élisabeth Privey, 7 avril 2014.

38. François Privey n'a pas fait d'études du fait de la guerre, mais aurait dû intégrer l'école d'ingénieur Marius Berliet à Lyon. C'était un homme cultivé.

39. Entretien de l'auteure avec Élisabeth Privey, 19 mars 2018.

40. Entretien de l'auteure avec Wanda Starzynska, août 2017 à Prignano sulla Secchia (Italie).

41. Wanda précise qu'elles rendent visite aux Polonaises Gaberlé, Sawka et Szyszkowski, les deux premières sont des anciennes collègues d'Andrzej.

42. Entretien de l'auteure avec Wanda Starzynska, août 2017, op. cit.

43. Entretien de l'auteure avec Élisabeth Privey, 19 mars 2018.

\section{RÉSUMÉS}

Au travers de témoignages, d'archives administratives et familiales, cet article interroge les stratégies de survie et de vie de deux femmes en migration dans un contexte de guerre et de sortie de guerre. Aldona Starzynska et Irena Borsukowska, toutes deux ressortissantes polonaises issues de l'intelligentsia, se sont rencontrées à Lyon. L'hypothèse de départ postulait qu'une solidarité de classe les avait rapprochées, mais le recours aux témoignages a largement nuancé et enrichi cette hypothèse. Il s'agit davantage de solidarité d'exil, de déracinement, basé sur ce que Thomas Kühne appelle le « social bonding ».

With the use of oral accounts, administrative and family archives, this paper explores within a context of war and end of war, the survival strategies of two women in migration. Polish nationals Aldona Starzynska and Irena Borsukowska, issued from the Polish Intelligentsia met in Lyon. The premise was that a class solidarity could explain their connexion, but recourse to 
oral accounts strongly qualified this idea. It was more a question of uprooting and solidarity in exile, based on Thomas Kühne concept of social bonding.

\section{INDEX}

Mots-clés : femmes en migration, stratégies de vie, Seconde Guerre mondiale, femmes polonaises, exil, rapatriement

Keywords : women in migration, life strategies, Second World War, Polish women, exile, repatriation

\section{AUTEUR}

\section{LAURENCE PREMPAIN}

Docteure en histoire de l'université Lyon 2, Laurence Prempain est chercheure associée au LARHRA (CNRS-UMR 5190), au CCFEF de l'université de Varsovie et membre du réseau Mémorha. Elle s'intéresse à différents aspects de la présence des Polonais.e.s et Juif.ve.s polonais.es en France de l'entre-deux-guerres à la fin de la Seconde Guerre mondiale (comme les réfugiée's, les migrations étudiantes, la persécution des Juif·ve·s et la Résistance polonaise en France). 